\title{
Salivary melatonin levels and sleep-wake rhythms in pregnant women with hypertensive and glucose metabolic disorders: A prospective analysis
}

\author{
Mieko Shimada $^{1, *}$, Hiroyuki Seki ${ }^{2}$, Michikazu Samejima ${ }^{3}$, Mako Hayase ${ }^{1}$, Fumie Shirai ${ }^{1}$ \\ 'Division of Health Sciences, Osaka University Graduate School of Medicine, Suita, Osaka, Japan; \\ ${ }^{2}$ Center for Maternal, Fetal and Neonatal Medicine, Saitama Medical Center, Saitama Medical University, Kawagoe, Saitama, Japan; \\ ${ }^{3}$ School of Nursing, Seirei Cristopher University, Hamamatsu, Shizuoka, Japan.
}

\begin{abstract}
Summary In preeclampsia and gestational diabetes, the sympathetic nerves are activated, leading to disrupted sleep. Melatonin, which transmits information to regulate the sleep-wake rhythm and other such biorhythms, has been implicated in insulin resistance, antioxidant behaviors, and metabolic syndrome. In addition, its reduced secretion increases the risk of hypertension and diabetes. The aim of this study was to elucidate the features of melatonin secretion, sleep quality, and sleep-wake rhythms in pregnant women with complications. Fifty-eight pregnant women with pregnancy complications (hypertensive or glucose metabolic disorders) and 40 healthy pregnant women completed questionnaires, including sleep logs and the Pittsburgh Sleep Quality Index (PSQI), during the second to third trimesters. Their salivary melatonin levels were also measured. Pregnant women with complications had significantly lower morning $(p<0.001)$, daytime $(p<0.01)$, evening $(p$ $<0.001)$, night $(p<0.01)$, daily mean $(p<0.001)$, peak $(p<0.001)$, and bottom $(p<0.01)$ melatonin values than healthy pregnant women. Pregnant women with complications also had significantly smaller melatonin amplitudes than healthy pregnant women $(p<0.001)$. Among pregnant women with complications, the duration $(p<0.05)$ and frequency $(p<$ 0.01) of wake after sleep-onset were significantly greater in the poor sleep group than in the favorable sleep group which was divided by PSQI cutoff value. Pregnant women with hypertensive or glucose metabolic disorder complications had smaller circadian variation in salivary melatonin secretion, and their values were lower throughout the day than healthy pregnant women.
\end{abstract}

Keywords: Melatonin, preeclampsia, gestational hypertension, gestational diabetes mellitus, sleep-wake rhythm, sleep quality

\section{Introduction}

The incidence of pregnancy at an advanced maternal age is continuously increasing; however, it is associated with an increased risk of pregnancy-induced hypertension (1) and an increased incidence of gestational diabetes

Released online in J-STAGE as advance publication February 6, 2016

*Address correspondence to:

Dr. Mieko Shimada, Division of Health Sciences, Osaka University Graduate School of Medicine. 1-7 Yamadaoka, Suita, Osaka 565-0871, Japan.

E-mail: shimadam@sahs.med.osaka-u.ac.jp mellitus (GDM) (2). Under the conditions of preeclampsia $(3,4)$ and GDM $(5)$, the sympathetic nerves are activated. Moreover, patients with preeclampsia often experience sleep disruptions due to an increased frequency of movement during rapid eye movement sleep ( 6 ) and those with diabetes tend to have difficulty falling asleep (7).

Exposure to light at night due to an irregular sleep schedule increases the incidence of elevated blood pressure (8) and pregnancy complications (9); therefore, a regular sleep rhythm and quality of sleep are crucial issues during pregnancy. Melatonin, a neuroendocrine hormone secreted from the pineal gland (10), reaches maximum secretion levels 3-5 h after usual sleep- 
onset (11) and decreases after awakening or upon light exposure (12). Melatonin is thus a humoral factor that transmits information to maintain biorhythms, such as the sleep-wake rhythm (13). Inhibited melatonin secretion in adult humans has also been associated with the incidence of obesity, metabolic syndrome (14), insulin resistance (15), and antioxidant activity (16). Experiments with rats have shown that melatonin stimulates insulin output and decreases free fatty acid synthesis (17). In addition, a large-scale survey of adult women demonstrated an association between low melatonin production and hypertension risk (18). Furthermore, the risk of developing diabetes has been reported to be higher among adults when melatonin secretion is low (19). However, no previous studies have assessed the association between melatonin secretion and the sleep-wake rhythm in pregnant women with certain complications. Thus, the aim of this study was to elucidate the features of melatonin secretion, sleep quality, and the sleep-wake rhythm in pregnant women with hypertensive or glucose metabolic disorders.

\section{Methods}

\subsection{Subjects and diagnostic criteria}

This study included 58 pregnant women who were diagnosed with a pregnancy-related complication (hypertensive or glucose metabolic disorder) in the second trimester ( $n=35$; week 16 day 0 to week 27 day 6 ) or third trimester ( $n=23$; week 28 day 0 to week 40 day 6). Patients were diagnosed using specified diagnostic criteria by a hospital obstetrician $(20,21)$. All patients received prenatal checkups between February 2009 and April 2012 at a university hospital in the Saitama prefecture and provided informed consent to participate in the present study. Among the 35 women who were diagnosed in the second trimester and participated in the present study, 20 were continuously surveyed until the third trimester. Forty healthy pregnant women in the third trimester (week 28 day 0 to week 40 day 6), who received prenatal checkups between August 2006 and April 2008 at a hospital in Osaka Prefecture, were included as controls and each also provided informed consent to participate in the present study.

The diagnostic criteria for hypertensive disorders included conditions of preeclampsia, gestational hypertension, and chronic hypertension. Glucose metabolic disorders included diabetes mellitus and gestational diabetes mellitus (GDM), which was diagnosed according to the criteria revised in 2010.

\subsection{Survey methods}

Although the onset of pregnancy complications typically occur in the third trimester, the survey was initiated in the second trimester because severe cases develop from the second trimester and often result in delivery before the third trimester. Surveys were administered to the healthy control subjects in the third trimester. All participants who gave informed consent for the study filled out a self-administered questionnaire and took saliva samples themselves at home, and sent these to us by mail. They were required to complete a questionnaire describing their characteristics and lifestyles, the Pittsburgh Sleep Quality Index (PSQI), and sleep logs. Saliva samples were collected for measuring melatonin levels. Information on diagnoses and treatment courses were collected from medical records, as was medical information relating to the type of delivery, gestational weeks, birth weight, delivery abnormalities, and postpartum course.

\subsection{Sleep logs}

Sleep logs are used as a form of auxiliary diagnosis in the field of psychiatry and enable ascertainment of life rhythms (22). In the present study, sleep logs, in which the subjects filled in their sleep time every $30 \mathrm{~min}$, were recorded daily for 1 week. Using a sleep-wake rhythm analysis program for Windows Ver. 3.0 (IAC, Tokyo, Japan), we analyzed sleep parameters that included total sleep time (total sleeping hours per day), nocturnal sleep time (sleeping hours per night), the duration of wake after sleep-onset [waking hours in the night (WASO)], the frequency of WASO, longest sleep time (LST), and sleep-onset time for LST.

\subsection{Pittsburgh Sleep Quality Index (PSQI)}

PSQI is a self-administered questionnaire (Likert scale of 0 to 3 ) developed by the University of Pittsburgh, Department of Psychiatry (Pittsburg, PA, USA) for assessing sleep quality (23). A Japanese language edition of PSQI was created by Doi et al. (24). The following seven components were scored: sleep quality (C1), sleep latency (C2), sleep duration (C3), habitual sleep efficiency $(\mathrm{C} 4$, the proportion of hours spent asleep in bed), sleep disturbance (C5), the use of sleeping medication (C6), and daytime dysfunction (C7). These elements were scored from 0 to 3 , and the scores were added together to calculate a total PSQI global score (PSQIG, 0-21). For all items, the higher the score, the more sleep was determined to have been disrupted. The cut-off point for the total score was 5.5 points $(23,24)$. Patients with a PSQIG $<5.5$ points were included in the favorable sleep group, and those with a PSQIG $\geq 5.5$ points were included in the poor sleep group.

\subsection{Measurement of melatonin concentration}

Saliva was collected at home 4 times daily for 3 days: before breakfast (6-8 a.m.), lunch (11 a.m.-1 p.m.), 
dinner (6-8 p.m.), and before going to sleep. At least 1.0 $\mathrm{mL}$ of saliva was collected in a 1.5-mL Safe-Lock Tube (Eppendorf, Hamburg, Germany) and immediately frozen at $-20^{\circ} \mathrm{C}$. Diurnal variation, acrophase (time of peak), and the amplitude of melatonin levels were analyzed. The melatonin concentration of the supernatant of the thawed and centrifuged (1500 $\mathrm{rpm}, 15 \mathrm{~min}, 4^{\circ} \mathrm{C}$ ) saliva was measured using a radioimmunoassay, as described by Samejima et al. (25), in which the measurement technique described by Miles et al. was modified (26). Measurements were made in duplicate using the following procedure. Melatonin antibodies (UK Code No. AB/S/01; Stockgrand, Ltd., Surrey, UK) were diluted 6000-fold with $0.1 \mathrm{M}$ tricine ( $\mathrm{pH}$ 7.5) (Sigma-Aldrich Corp., St. Louis, MO, USA) buffered with $0.9 \% \mathrm{NaCl}$ and $0.1 \%$ gelatin (Sigma-Aldrich Japan K.K., Tokyo, Japan). For a standard solution of melatonin, melatonin powder (M5250-1G, Code No. M5259; SigmaAldrich Corp.) was used. For the radioimmunoassay, $\left[{ }^{3} \mathrm{H}\right]$-melatonin (GE Healthcare Bio-Sciences, Tokyo, Japan) was used with charcoal (242276-250G activated charcoal DARCO; Sigma-Aldrich Corp.), DextranT-70 (Extrasynthese SA, Genay, France), and scintillation cocktail (Hionic-Fluor; PerkinElmer, Inc., Waltham, MA, USA). All procedures were performed in duplicate. The intra- and inter-assay coefficients of variation in the experiments were $3.5 \%$ and $10.7 \%$, respectively.

\subsection{Statistical analysis}

We used an unpaired t-test to compare mean values between the two groups, a paired t-test to compare mean values for continuous cases from the second to third trimester, the $\chi^{2}$ test to compare frequency, oneway analysis of variance to confirm circadian variation in the two groups, two-way analysis of variance to compare circadian variation, and Pearson's correlation coefficient to analyze relationships between each of the sleep indicators. All statistical analyses were performed using SPSS J for Windows ver. 22.0 (IBM-SPSS, Inc., Chicago, IL, USA).

\subsection{Ethical Considerations}

The study protocol was approved by the ethics committees of the Osaka University Graduate School of Medicine, Osaka, Japan and the Saitama Medical University, Saitama, Japan.

\section{Results}

\subsection{Subject attributes}

The ages (mean $35.1 \pm 4.2 \mathrm{SD}$ ), gestational weeks at delivery (mean $38.0 \pm 1.3 \mathrm{SD}$ ), infant weight (mean $2898 \pm 434 \mathrm{SD}$ ), complications (Hypertensive disorder 31, Glucose metabolism disorder 19, both 8), treatments, and outcomes upon delivery of the study subjects are shown in Table 1.

\subsection{Circadian variation in salivary melatonin concentration}

For both pregnant women with complications in either the second or third trimester and healthy pregnant women, melatonin levels were the highest at night, decreased throughout the early morning, and were the lowest during the day, demonstrating significant daily fluctuations $(p<0.001)$ (Figure 1). Pregnant women with complications had significantly lower morning ( $p<0.001)$, daytime $(p<0.01)$, evening ( $p<$ $0.001)$, night-time $(p<0.01)$, daily mean $(p<0.001)$, peak $(p<0.001)$, and bottom $(p<0.01)$ melatonin levels than healthy pregnant women (Figure 1). The amplitude, or the difference between the highest and lowest melatonin values in one day, was significantly smaller for pregnant women with complications than that for healthy pregnant women $(p<0.001)$. Among pregnant women with complications, the times of peak $(p<0.05)$ and bottom $(p<0.05)$ melatonin levels were significantly later in the third trimester than those in the second trimester (Table 2).

\subsection{Sleep parameters in pregnant women}

There were no significant differences in the analyzed sleep parameters between pregnant women with complications and healthy pregnant women in the third trimester. However, pregnant women with complications had significantly later sleep-onset times $(p<0.01)$, shorter LST $(p<0.01)$, and shorter nocturnal sleep times $(p<0.01)$ in the third trimester than those in the second trimester (Table 3).

\subsection{Comparison of sleep indicators according to PSQI}

Among the pregnant women with complications, 15 (42.9\%) were in the poor sleep group (PSQIG score $\geq$ $5.5)$ in the second trimester and $23(53.5 \%)$ were in the poor sleep group in the third trimester, while 20 women $(50.0 \%)$ in the control group were in the poor sleep group during the third trimester. No significant difference in the frequency of poor sleep group and the mean value by PSQI, was observed between pregnant women with complications and healthy pregnant woman in the third trimester. Among pregnant women with complications, the duration and frequency of WASO of the sleep logs were significantly greater in the PSQI poor sleep group than those in the favorable sleep quality group in the second trimester ( $p<0.05$ and $p<0.01$, respectively). In the third trimester, sleep-onset times were significantly later in the poor sleep group than those in the favorable sleep quality group $(p<0.05)$. 
Table 1. Demographic characteristics

\begin{tabular}{|c|c|c|c|c|c|c|c|c|c|c|}
\hline \multirow{2}{*}{ Items } & \multicolumn{5}{|c|}{ Pregnant women with complications $(n=58)$} & \multicolumn{5}{|c|}{ Healthy pregnant women $(n=40)$} \\
\hline & Mean & SD & Range & $n$ & $\%$ & Mean & SD & Range & $n$ & $\%$ \\
\hline Age (years) & 35.1 & 4.2 & $27-45$ & & & 33.3 & 4.3 & $27-43$ & & \\
\hline Height $(\mathrm{cm})$ & 157.4 & 6.3 & $142-170$ & & & - & & & & \\
\hline Non-pregnant weight $(\mathrm{kg})$ & 66.8 & 17.1 & $40.0-106.6$ & & & - & & & & \\
\hline Non-pregnant BMI (\%) & 26.8 & 6.2 & $18.4-41.7$ & & & - & & & & \\
\hline Weight increase (kg) & 6.8 & 5.2 & -6.8 to 18.0 & & & - & & & & \\
\hline Infant weight (g) & 2898.3 & 434.0 & $1855-4168$ & & & 3048.2 & 403.6 & $2235-3880$ & & \\
\hline Gestational week (weeks) & 38.0 & 1.3 & $34-40$ & & & 39.0 & 1.5 & $35-42$ & & \\
\hline \multicolumn{11}{|l|}{ Employment } \\
\hline Yes & & & & 22 & 37.9 & & & & 15 & 37.5 \\
\hline No & & & & 34 & 58.6 & & & & 25 & 62.5 \\
\hline Unknown & & & & 2 & 3.4 & & & & & \\
\hline \multicolumn{11}{|l|}{ Parity } \\
\hline Primipara & & & & 32 & 55.2 & & & & 34 & 85.0 \\
\hline Multipara & & & & 26 & 44.8 & & & & 6 & 15.0 \\
\hline \multicolumn{11}{|l|}{ Complications } \\
\hline \multicolumn{11}{|l|}{ Hypertensive disorder } \\
\hline Preeclampsia & & & & 5 & 8.6 & & & & - & \\
\hline GH & & & & 5 & 8.6 & & & & - & \\
\hline HT & & & & 21 & 36.2 & & & & - & \\
\hline \multicolumn{11}{|c|}{ Glucose metabolism disorder } \\
\hline GDM & & & & 19 & 32.9 & & & & - & \\
\hline \multicolumn{11}{|l|}{ Both } \\
\hline $\mathrm{HT}+\mathrm{GDM}$ & & & & 6 & 10.3 & & & & - & \\
\hline $\mathrm{GH}+\mathrm{GDM}$ & & & & 1 & 1.7 & & & & - & \\
\hline $\mathrm{GH}+\mathrm{DM}$ & & & & 1 & 1.7 & & & & - & \\
\hline \multicolumn{11}{|l|}{ Treatment during pregnancy } \\
\hline Yes & & & & 41 & 70.7 & & & & - & \\
\hline No & & & & 17 & 29.3 & & & & - & \\
\hline \multicolumn{11}{|l|}{ Type of treatment } \\
\hline Dietary advice & & & & 7 & 17.1 & & & & - & \\
\hline Oral medicine & & & & 18 & 43.9 & & & & - & \\
\hline Infusion or injections & & & & 16 & 39.0 & & & & - & \\
\hline \multicolumn{11}{|l|}{ Delivery type } \\
\hline Normal delivery & & & & 25 & 43.1 & & & & 26 & 65.0 \\
\hline Caesarean section & & & & 29 & 50.0 & & & & 13 & 32.5 \\
\hline Forceps delivery & & & & 4 & 6.9 & & & & 0 & 0.0 \\
\hline Unknown & & & & & & & & & 1 & 2.5 \\
\hline \multicolumn{11}{|l|}{ Postpartum treatment } \\
\hline Yes & & & & 25 & 43.1 & & & & - & \\
\hline No & & & & 33 & 56.9 & & & & - & \\
\hline
\end{tabular}

SD, standard deviation; BMI, body mass index; GH, gestational hypertension; HT, chronic hypertension; GDM, gestational diabetes mellitus; $\mathrm{DM}$, diabetes mellitus

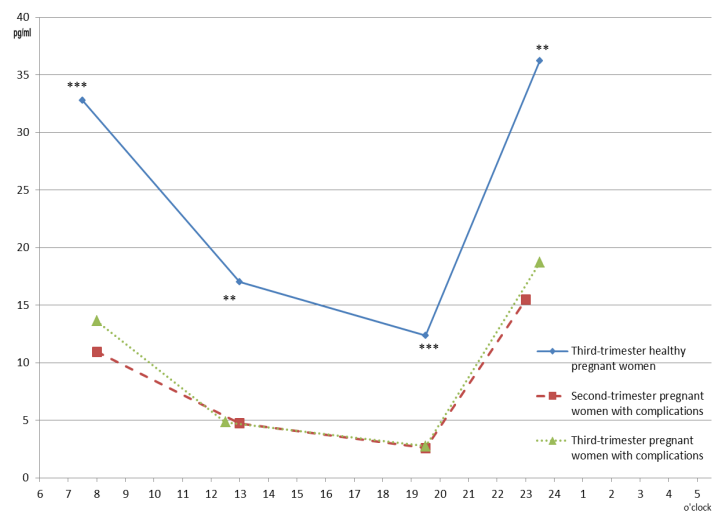

Figure 1. Circadian changes in salivary melatonin levels. Salivary melatonin levels in pregnant women with complications were significantly lower than those in healthy pregnant women at all times (morning, daytime, evening, and nighttime). ${ }^{* * *} p<$ $0.001,{ }^{* *} p<0.01$

\subsection{Correlation between melatonin values and sleep indicators}

PSQI sleep efficiency (C4) for healthy pregnant women in the third trimester showed a negative correlation with night-time $(r=-0.388, p<0.05)$, peak $(r=$ $-0.324, p<0.05)$, and amplitude $(r=-0.324, p<0.05)$ melatonin values. However, in pregnant women with complications, these correlations were not observed in either the second or third trimester.

\section{Discussion}

This is the first study to analyze long-term melatonin secretion, sleep-wake rhythms, and sleep quality in pregnant women with hypertensive or glucose 
Table 2. Melatonin indicators

\begin{tabular}{|c|c|c|c|c|c|c|c|}
\hline \multirow{2}{*}{ Items } & \multicolumn{2}{|c|}{$\begin{array}{l}\text { Second-trimester pregnant } \\
\text { women with complications } \\
\qquad(n=35)\end{array}$} & \multicolumn{2}{|c|}{$\begin{array}{l}\text { Third-trimester pregnant } \\
\text { women with complications } \\
\qquad(n=43)\end{array}$} & \multicolumn{2}{|c|}{$\begin{array}{l}\text { Third-trimester healthy } \\
\text { pregnant women } \\
(n=40)\end{array}$} & \multirow{2}{*}{$p$-value } \\
\hline & Mean & SE & Mean & SE & Mean & SE & \\
\hline Daily mean melatonin $(\mathrm{pg} / \mathrm{mL})^{\dagger}$ & 8.4 & 1.2 & 10.2 & 1.4 & 23.8 & 2.6 & $<0.001$ \\
\hline Peak value $(\mathrm{pg} / \mathrm{mL})^{\dagger}$ & 20.1 & 2.8 & 22.5 & 2.9 & 46.4 & 4.4 & $<0.001$ \\
\hline Bottom value $(\mathrm{pg} / \mathrm{mL})^{\dagger}$ & 1.7 & 0.4 & 2.4 & 0.5 & 7.3 & 1.3 & $<0.01$ \\
\hline Amplitude $(\mathrm{pg} / \mathrm{mL})^{\dagger}$ & 18.4 & 2.6 & 20.0 & 2.6 & 39.2 & 3.8 & $<0.001$ \\
\hline Peak value time (o'clock $)^{*}$ & 24.2 & 0.9 & 27.0 & 0.7 & 25.6 & 1.0 & $<0.05$ \\
\hline Bottom value time (o'clock) & 15.6 & 0.7 & 17.7 & 0.6 & 16.9 & 0.7 & $<0.05$ \\
\hline
\end{tabular}

Comparison between third-trimester pregnant women with complications and healthy pregnant women using the unpaired $t$-test. ${ }^{\ddagger}$ Comparison between second- and third-trimester pregnant women with complications using the unpaired $t$-test. SE, standard error.

Table 3. Sleep log indicators

\begin{tabular}{|c|c|c|c|c|c|c|c|}
\hline \multirow{2}{*}{ Items } & \multicolumn{2}{|c|}{$\begin{array}{l}\text { Second-trimester pregnant } \\
\text { women with complications } \\
\qquad(n=35)\end{array}$} & \multicolumn{2}{|c|}{$\begin{array}{l}\text { Third-trimester pregnant } \\
\text { women with complications } \\
\qquad(n=43)\end{array}$} & \multirow{2}{*}{$p$-value } & \multicolumn{2}{|c|}{$\begin{array}{l}\text { Third-trimester healthy } \\
\text { pregnant women } \\
(n=40)\end{array}$} \\
\hline & Mean & $\mathrm{SE}$ & Mean & SE & & Mean & SE \\
\hline Total sleep time $(\mathrm{h})$ & 8.1 & 0.2 & 7.6 & 0.2 & NS & 7.8 & 0.2 \\
\hline Nighttime sleep time (h) & 7.2 & 0.2 & 6.5 & 0.2 & $<0.01$ & 6.8 & 0.2 \\
\hline Duration of wake after sleep-onset (min) & 20.4 & 4.6 & 25.2 & 5.8 & NS & 25.4 & 6.9 \\
\hline Frequency of WASO (times) & 0.2 & 0.0 & 0.3 & 0.1 & NS & 0.3 & 0.1 \\
\hline Longest sleep time (h) & 7.1 & 0.2 & 6.2 & 0.2 & $<0.01$ & 6.6 & 0.2 \\
\hline Sleep-onset time for LST (o'clock) & 23.7 & 0.2 & 24.5 & 0.2 & $<0.01$ & 24.4 & 0.2 \\
\hline
\end{tabular}

Comparison between the second- and third-trimester pregnant women with complications using the unpaired $t$-test. Comparison between the third-trimester pregnant women with complications and healthy pregnant women using the unpaired $t$-test. SE, standard error; WASO, waking hours after sleep onset; LST, longest sleep time; NS, not significant

metabolic disorders from the second to third trimester.

We measured salivary melatonin levels as a physiological indicator of sleep. Serum melatonin levels fluctuate throughout the day and peak at night (27). In the present study, we measured melatonin using saliva samples rather than blood samples because the salivary melatonin assay measured biologically active melatonin, since only free melatonin passes through the parotid membrane. Moreover, the collection of saliva is easier and less invasive than blood sampling at home.

Our study first elucidated that pregnant women with hypertensive or glucose metabolic disorder complications had lower melatonin secretion throughout the day (morning, daytime, evening, and night-time) than healthy pregnant women in both the second and third trimesters. Similar results were obtained with daily mean, peak, and bottom melatonin levels, indicating that pregnant women with complications have inhibited melatonin secretion throughout the day by measuring circadian variation in salivary melatonin secretion four times a day.

Melatonin is a humoral factor that controls the circadian rhythm of living organisms over approximately 24 hour cycles including the sleep-wake cycle, with high levels of melatonin observed after entering sleep at night. Insulin concentration, which adjusts blood glucose levels and blood pressure varies during this 24-hour cycle, with low levels reached at night. There are three types of melatonin receptors, comprising MT1, MT2 and MT3 receptors. When MT2 receptors bind with melatonin, this results in adjustment to the biological clock. There are also melatonin receptors present in the beta cells of the pancreas. MT2 receptors in beta cells of the pancreas are involved in insulin secretion adjustment as they block cyclic adenosine monophosphate (cAMP), an important metabolism signal that controls insulin secretion, and induce the formation of cyclic guanosine monophosphate (cGMP) (28).

However, genetic mutation in the MT2 receptors prevents them from responding to melatonin, thereby blocking insulin release from beta cells in the pancreas and increasing risk for type 2 diabetes. This has been confirmed by genetic analysis of approximately 12,000 subjects (29). Blood pressure exhibits diurnal variation over a 24-hour cycle mainly due to autonomic nervous action. Various factors are related to hypertension. These are thought to include, in addition to excessive sodium intake and autonomic nervous irregularity (30), disturbance of the biological clock and decreased melatonin secretion (31). Melatonin, for which high levels are observed at night during sleep, causes vasodilation, suppression of the sympathetic nervous system and hypotension (32). Melatonin increases cytoplasmic calcium and nitric monoxide in endothelial cells, thereby increasing vasodilation lowering the serum norepinephrine level. Therefore, low levels 
of melatonin may be an onset factor for pregnancy complications, which exhibit pathophysiology that is similar to type 2 diabetes and hypertension (33).

Melatonin reportedly protects cellular apoptosis in the placenta by acting as an antioxidant (34) and a free radical scavenger (35). Melatonin also inhibits the vasospasticity of the umbilical artery (36), and when administered, possibly prevents the risk of preeclampsia (37). These findings suggest that the risk of preeclampsia is higher when melatonin secretion is decreased. Meanwhile, when melatonin secretion is reduced, the action of insulin receptors is negatively affected in adults (38), thereby increasing insulin resistance in non-pregnant women (15). Therefore, there is an increased risk of diabetes when melatonin secretion is reduced, which presumably also increases the risk of gestational diabetes in pregnant women.

In the present study, we observed later times of peak and bottom melatonin values in the third trimester compared with those in the second trimester; melatonin secretion rhythm lagged as a result of the significant delay in third trimester sleep-onset times. The amplitude in salivary melatonin secretion was decreased in pregnant women with complications in the third trimester in the present study and was likely because melatonin decreases after awaking, upon light exposure (12), or when sleep is shallow (39), and the sleep state often becomes shallow by polysomnography in the third trimester (40).

Healthy pregnant women with greater sleep efficiency had longer sleep times at night and greater melatonin peak values and amplitude. However, this trend was not observed in pregnant women with complications, as this group demonstrated shorter nocturnal sleep times and LST in the third trimester. This may have been because the sympathetic nervous system is dominant in women with gestational diabetes and preeclampsia $(4,30)$, meaning that secretion of the sleep-inducing substance melatonin at night, when the parasympathetic nervous system becomes dominant, was lower in pregnant women with complications than in healthy pregnant women in this study. This meant that they had poorer quality sleep and that healthy sleep efficiency and nighttime sleeping were impaired. Poor sleep efficiency, a PSQI component, due to arousal during sleep or difficulty falling asleep resulted in decreased melatonin secretion. However, melatonin secretion in pregnant women has been reported to correlate with melatonin secretion in cord blood (41) and to impact the production of fetal melatonin receptors through the placental stage (42), suggesting that melatonin secretion in pregnant women impacts the sleep-wake rhythm in infants after birth.

Therefore, promoting ample melatonin secretion by adjusting the sleep-wake rhythm during pregnancy and improving sleep quality is important not only to prevent hypertensive and glucose metabolic disorders but also to develop the sleep-wake rhythm in the fetus. Enhancing melatonin secretion requires strategies to increase sleep efficiency. For example, we recommend the following to improve sleep efficiency: darkening the room early at night (12), bathing before bed because body temperature and melatonin secretion are related (43), going to the toilet before bed to reduce WASO caused by frequent urination (40), going to bed early to increase sleeping hours (44), and maintaining a regular daily life rhythm.

There were certain limitations to the present study that should be addressed. First, we could not clarify the features of melatonin secretion associated with each diagnosis (hypertension and diabetes) because of the small number of subjects. In addition, the present study did not include matched controls because the control group only included healthy pregnant women in the third trimester.

\section{Conclusion}

Pregnant women with hypertensive or glucose metabolic disorders had smaller circadian variation in salivary melatonin secretion, and their melatonin values were lower throughout the day than healthy pregnant women. Moreover, pregnant women with these complications exhibited shorter nocturnal sleep times and LST. Overall, the sleep quality of both pregnant women with complications and healthy pregnant women was worse compared with that of non-pregnant women.

\section{Acknowledgements}

The authors thank Mizue Matsumoto, RM, head midwife, and her colleagues at the Center for Maternal, Fetal and Neonatal Medicine, Saitama Medical Center, Saitama Medical University, for assisting with data collection. This study was funded by a 20042007 Grant-in-Aid for Scientific Research B from the Ministry of Education, Culture, Sports, Science and Technology, Japan (No. 16390638) and a 20082012 Grant-in-Aid for Scientific Research B from the Ministry of Education, Culture, Sports, Science and Technology, Japan (No. 20390562), both awarded to Mieko Shimada.

\section{References}

1. Roach VJ, Hin LY, Tam WH, Ng KB, Rogers MS. The incidence of pregnancy-induced hypertension among patients with carbohydrate intolerance. Hypertens Pregnancy. 2000; 19:183-189.

2. Morikawa M, Yamada T, Yamada T, Sato S, Cho K, Minakami H. Prevalence of hyperglycemia during pregnancy according to maternal age and pre-pregnancy body mass index in Japan, 2007-2009. Int J Gynaecol Obstet. 2012; 118:198-201.

3. Fisher T, Schobel HP, Frank H, Andreae M, Schneider 
KT, Heusser K Pregnancy-induced sympathetic overactivity: A precursor of preeclampsia. Eur J Clin Invest. 2004, 34:443-448.

4. Yang CH, Chao TC, Kuo BT, Yin CS, Chen HS Preeclamptic pregnancy is associated with increased sympathetic and decreased parasympathetic control of HR. Am J Physiol Heart Circ Physiol. 2000; 278:H1269-H1273.

5. Weissman A, Lowenstein L, Peleg A, Thaler I, Zimmer EZ. Power spectral analysis of heart rate variability during the 100-g oral glucose tolerance test in pregnant women. Diabetes Care. 2006; 29:571-574.

6. Ekholm EM, Polo O, Rauhala ER, Ekblad UU. Sleep quality in preeclampsia. Am J Obstet Gynecol. 1992; 167:1262-1266.

7. Sridhar GR, Madhu K. Prevalence of sleep disturbances in diabetes mellitus. Diabetes Res Clin Pract. 1994; 23:183-186.

8. Simko F, Reiter RJ, Pechanova O, Paulis L. Experimental models of melatonin-deficient hypertension. Front Biosci (Landmark Ed). 2013; 18:616-625.

9. Tamura H, Nakamura Y, Terron MP, Flores LJ, Manchester LC, Tan DX, Sugino N, Reiter RJ. Melatonin and pregnancy in the human. Reprod Toxicol. 2008; 25:291-303.

10. Reiter RJ. Pineal melatonin: cell biology of its synthesis and of its physiological interactions. Endocr Rev. 1991; 12:151-180.

11. Waldhauser F, Dietzel M. Daily and annual rhythms in human melatonin secretion: role in puberty control. Ann N Y Acad Sci. 1985; 453:205-214

12. Weinberg U, D'Eletto RD, Weitzman ED, Erlich S, Hollander CS. Circulating melatonin in man: episodic secretion throughout the light-dark cycle. J Clin Endocrinol Metab. 1979; 48:114-118.

13. Reppert SM, Sagar SM. Characterization of the daynight variation of retinal melatonin content in the chick. Invest Ophthalmol Vis Sci. 1983; 24:294-300.

14. Reiter RJ, Tan DX, Korkmaz A, Ma S. Obesity and metabolic syndrome: association with chronodisruption, sleep deprivation, and melatonin suppression. Ann Med. 2012; 44:564-577.

15. McMullan CJ, Curhan GC, Schernhammer ES, Forman JP. Association of nocturnal melatonin secretion with insulin resistance in nondiabetic young women. Am J Epidemiol. 2013; 178:231-238.

16. Aversa S, Pellegrino S, Barberi I, Reiter RJ, Gitto E. Potential utility of melatonin as an antioxidant during pregnancy and in the perinatal period. J Matern Fetal Neonatal Med. 2012; 25:207-221.

17. Fabiś M, Pruszyńska E, Maćkowiak P. In vivo and in situ action of melatonin on insulin secretion and some metabolic implications in the rat. Pancreas. 2002; 25:166-169.

18. Forman JP, Curhan GC, Schernhammer ES. Urinary melatonin and risk of incident hypertension among young women. J Hypertens. 2010; 28:446-451.

19. McMullan CJ, Schernhammer ES, Rimm EB, Hu FB, Forman JP. Melatonin secretion and the incidence of type 2 diabetes. JAMA. 2013; 309:1388-1396.

20. Seki H.The role of the renin-angiotensin system in the pathogenesis of preeclampsia - new insights into the renin-angiotensin system in preeclampsia. Med Hypotheses. 2014; 82:362-367.

21. Guidelines for office gynecology in Japan: Japan Society of Obstetrics and Gynecology and Japan Association of Obstetricians and Gynecologists 2011 edition. 2011; 3-4 http://www.jsog.or.jp/activity/pdf/gl_obst_2011_en.pdf (accessed May 1, 2014)

22. Okawa M, Uchiyama M, Ozaki S, Shibui K, Kamei Y, Hayakawa T, Urata J. Melatonin treatment for circadian rhythm sleep disorders. Psychiatry Clin Neurosci. 1998; 52:259-260.

23. Buysse DJ, Reynolds CF 3rd, Monk TH, Berman SR, Kupfer DJ. The Pittsburgh Sleep Quality Index: A new instrument for psychiatric practice and research. Psychiatry Res. 1989; 28:193-213.

24. Doi Y, Minowa M, Uchiyama M, Okawa M, Kim K, Shibui K, Kamei Y. Psychometric assessment of subjective sleep quality using the Japanese version of the Pittsburgh Sleep Quality Index (PSQI-J) in psychiatric disordered and control subjects. Psychiatry Res. 2000; 97(2-3):165-172.

25. Samejima M, Shavali S, Tamotsu S, Uchida K, Morita Y, Fukuda A. Light- and temperature-dependence of the melatonin secretion rhythm in the pineal organ of the lamprey, Lampetra japonica. Jpn J Physiol. 2000; 50:437-442.

26. Miles A, Philbrick D, Tidmarsh SF, Shaw DM. Direct radioimmunoassay of melatonin in saliva. Clin Chem. $1985 ; 31: 1412-1413$.

27. Peschke E, Frese T, Chankiewitz E, Peschke D, Preiss U, Schneyer U, Spessert R, Mühlbauer E. Diabetic Goto Kakizaki Rats as well as type 2 diabetic patients show a decreased diurnal serum melatonin level and an increased pancreatic melatonin-receptor status. J Pineal Res. 2006; 40:135-143.

28. Peschke E, Bahr I, Mühlbauer Experimental and aspects of melatonin and clock genes in diabetes. J Pinel Res. 2015; 59:1-23.

29. Prokopenko I, Langenberg C, Florez $\mathrm{Cj}$, et al. Variants in MTNR1B influence fasting glucose levels. Nature Genetics. 2009; 41:77-81.

30. Metsaars WP, Ganzevoort W, Karemaker JM, Rang S, Wolf $\mathrm{H}$ Increased sympathetic activity present in early hypertensive pregnancy is not lowered by plasma volume expansion. Hypertens Pregnancy 2006; 25:143-157.

31. Pechanova O, Paulis L, Simko F Peripheral and Central Effects of Melatonin on Blood Pressure Regulation. Int J Mol Sci. 2014; 15:17920-17937.

32. Obayashi K, Saeki K, Tone N2, Kurumatani N Relationship between melatonin secretion and nighttime blood pressure in elderly individuals with and without antihypertensive treatment: a cross-sectional study of the HEIJO-KYO cohort. Hypertens Res. 2014; 37:908-913.

33. Arangino S, Cagnacci A, Angiolucci M, Vacca MA, Longu G, Volpe A, Melis GB. Effects of Melatonin on Vascular Reactivity, Catecholamin Levels, and Blood Pressure in Healthy Men. Am J Cardiol. 1999; 83:14171419.

34. Tamura H, Takasaki A, Taketani T, Tanabe M, Lee L, Tamura I, Maekawa R, Aasada H, Yamagata Y, Sugino N. Melatonin and female reproduction. J Obstet Gynaecol Res. 2014; 40:1-11

35. Reiter RJ, Tan DX, Poeggeler B, Menendez-Pelaez A, Chen LD, Saarela S. Melatonin as a free radical scavenger: Implications for aging and age-related diseases. Ann N Y Acad Sci. 1994; 719:1-12.

36. Okatani Y, Watanabe K, Hayashi K, Wakatsuki A, Sagara Y. Melatonin inhibits vasospastic action of hydrogen 
peroxide in human umbilical artery. J Pineal Res 1997; 22:163-168.

37. Lanoix D, Guérin P, Vaillancourt C. Placental melatonin production and melatonin receptor expression are altered in preeclampsia: new insights into the role of this hormone in pregnancy. J Pineal Res. 2012; 53:417-425.

38. Peschke E, Mühlbauer E. New evidence for a role of melatonin in glucose regulation. Best Pract Res Clin Endocrinol Metab. 2010; 24:829-841.

39. Mishima K, Okawa M, Shimizu T, Hishikawa Y. Diminished melatonin secretion in the elderly caused by insufficient environmental illumination. J Clin Endocrinol Metab. 2001; 86:129-134.

40. Hertz G, Fast A, Feinsilver SH, Albertario CL, Schulman H, Fein AM. Sleep in normal late pregnancy. Sleep. 1992; 15:246-251.

41. Okatani Y, Okamoto K, Hayashi K, Wakatsuki A, Tamura
S, Sagara Y. Maternal-fetal transfer of melatonin in pregnant women near term. J Pineal Res. 1998; 25:129134.

42. Reppert SM, Weaver DR, Rivkees SA, Stopa EG. Putative melatonin receptors in a human biological clock. Science. 1988; 242: 78-81.

43. Kubota T, Uchiyama M, Suzuki H, Shibui K, Kim K, Tan $\mathrm{X}$, Tagaya $\mathrm{H}$, Okawa $\mathrm{M}$, Inoue $\mathrm{S}$. Effects of nocturnal bright light on saliva melatonin, core body temperature and sleep propensity rhythms in human subjects. Neurosci Res. 2002; 42:115-122.

44. Tsai SY, Kuo LT, Lai YH, Lee CN. Factors associated with sleep quality in pregnant women: A prospective observational study. Nurs Res. 2011; 60:405-412.

(Received September 20, 2015; Revised January 26, 2016; Accepted January 28, 2016) 